\title{
Correction to: Tertiary Care Experience of Sorafenib in the Treatment of Progressive Radioiodine-Refractory Differentiated Thyroid Carcinoma: A Korean Multicenter Study, by Kim M, Kim TH, Shin DY, Lim DJ, Kim EY, Kim WB, Chung JH, Shong YK, Kim BH, and Kim WG on behalf of Korean Thyroid Cancer Study Group (KTCSG). Thyroid 2018;28(3)340-348. DOI: 10.1089/thy.2017.0356
}

\footnotetext{
N THE March 2018 issue of Thyroid (vol. 28, no.3; 340-348), the article entitled "Tertiary Care Experience of Sorafenib in the Treatment of Progressive Radioiodine-Refractory Differentiated Thyroid Carcinoma: A Korean Multicenter Study"' by Kim et al. requires correction.

The first two authors of this article, Mijin Kim and Tae Hyuk Kim, share co-first authorship. The authors should appear as:

Mijin Kim, ${ }^{1, *}$ Tae Hyuk Kim, ${ }^{2,}{ }^{*}$ Dong Yeob Shin, ${ }^{3}$ Dong Jun Lim, ${ }^{4}$

Eui Young Kim, ${ }^{5}$ Won Bae Kim, ${ }^{1}$ Jae Hoon Chung, ${ }^{2}$ Young Kee Shong, ${ }^{1}$

Bo Hyun Kim, ${ }^{6}$ and Won Gu $\mathrm{Kim}^{1}$ on behalf of Korean Thyroid Cancer Study Group (KTCSG)

${ }^{1}$ Division of Endocrinology and Metabolism, Department of Internal Medicine, Asan Medical Center, University of Ulsan College of Medicine, Seoul, Korea.

${ }^{2}$ Division of Endocrinology and Metabolism, Department of Medicine, Thyroid Center, Samsung Medical Center, Sungkyunkwan University School of Medicine, Seoul, Korea.

${ }^{3}$ Division of Endocrinology and Metabolism, Department of Internal Medicine, Severance Hospital, Yonsei University College of Medicine, Seoul, Korea.

${ }^{4}$ Division of Endocrinology and Metabolism, Department of Internal Medicine, Seoul St. Mary's Hospital, The Catholic University of Korea, Seoul, Korea.

${ }^{5}$ Department of Endocrinology, Dongnam Institute of Radiological and Medical Sciences Cancer Center, Busan, Korea.

${ }^{6}$ Division of Endocrinology and Metabolism, Department of Internal Medicine, Biomedical Research Institute, Pusan National University Hospital, Busan, Korea.

*Co-first authors.
}

The online version of the article has been corrected to reflect this change. 